$\begin{array}{ll} & \text { Etnográfica } \\ \text { etnográfica } & \text { Revista do Centro em Rede de Investigação em }\end{array}$

Antropologia

vol. 24 (1) | 2020

Vol. $24(1)$

\title{
Merecimiento y lenguajes de la injusticia: introducción
}

Mikel Aramburu Otazu y Irene Sabaté Muriel

\section{(2) OpenEdition}

Journals

Edición electrónica

URL: https://journals.openedition.org/etnografica/8326

DOI: 10.4000/etnografica.8326

ISSN: 2182-2891

\section{Editor}

Centro em Rede de Investigação em Antropologia

\section{Edición impresa}

Fecha de publicación: 1 febrero 2020

Paginación: 157-164

ISSN: 0873-6561

\section{Referencia electrónica}

Mikel Aramburu Otazu y Irene Sabaté Muriel, «Merecimiento y lenguajes de la injusticia: introducción», Etnográfica [En línea], vol. 24 (1) | 2020, Publicado el 25 febrero 2020, consultado el 20 enero 2022. URL: http://journals.openedition.org/etnografica/8326 ; DOI: https://doi.org/10.4000/etnografica.8326

\section{(c) (7) (8)}

Etnográfica is licensed under a Creative Commons Attribution-NonCommercial 4.0 International License. 


\section{Merecimiento y lenguajes de la injusticia: introducción}

Mikel Aramburu Otazu e Irene Sabaté Muriel

ARAMBURU OTAZU, Mikel (mikel.aramburu@ub.edu) - Departament d'Antropologia Social, Universitat de Barcelona, España.

SABATÉ MURIEL, Irene (sabate.irene@gmail.com) - Departament d'Antropologia Social, Universitat de Barcelona, España. 
EL CONCEPTO DE (UN)DESERVINGNESS SE REMONTA, EN EL ÁMBITO DE las ciencias sociales y la historiografía anglosajonas, a la tradición liberal que inspiró formas de gestión de la pobreza basadas en clasificaciones morales que gobernaban y justificaban la asignación de recursos por parte de un naciente Estado benefactor y de otras entidades de acción social. ${ }^{1}$

Más allá, sin embargo, de la ya tradicional distinción entre "pobres merecedores" y "pobres inmerecedores" (deserving and undeserving poor) (Katz 1989), el presente monográfico se propone dar cuenta de la utilidad de la noción de (in) merecimiento en un sentido analítico más amplio, entendida como una forma recurrente de expresar los sentidos de injusticia sobre la base de argumentos morales que sustentan o bien el ejercicio propio de derechos formales o entitlements, o bien la restricción del acceso a estos derechos por parte de otros.

\section{MERECIMIENTO Y ECONOMÍA MORAL DEL NEOLIBERALISMO}

Puede argumentarse que la ética del trabajo y del esfuerzo no es una novedad de nuestra época, sino que está presente en las sociedades desde la fundación del capitalismo, como también lo ha estado en experiencias históricas no capitalistas, como el socialismo real. En consecuencia, aquellos grupos sociales representados como improductivos, bajo distintas denominaciones (lumpen-proletariado, underclass o infraclase, undeserving poor o pobres indignos...), tienden a ser considerados como inmerecedores de reconocimiento y recursos. El debate social al respecto, que puede rastrearse muy hacia atrás en la historia del capitalismo (Ruggiero 2018), conoce un nuevo auge en la actual era neoliberal.

En efecto, la lógica del merecimiento se exacerba hoy de la mano de conceptos como el workfarism, y bajo los ideales de independencia, emprendeduría y responsabilidad individual. Algunos autores, como Whyte y Wiegratz (2016), proponen entender el conjunto de valores que constituyen este orden ideológico como la manifestación de una economía moral peculiar, la economía moral del neoliberalismo, que se contrapondría a la que en su día describieron E.P. Thompson (1971) y James Scott (1976), quienes acuñaron el término "economía moral" para designar formas de resistencia popular ante la implantación del mercado capitalista.

Como característica fundamental, el uso de la noción de merecimiento en el momento histórico presente se ubica en el contexto de una disminución de los recursos públicos, bajo unas políticas de austeridad legitimadas por la crisis

$1 \quad$ El presente monográfico es fruto de los debates desarrollados en el marco del proyecto "Concepciones Populares de la Justicia Social ante la Crisis y las Políticas de Austeridad” (CSO2015-67368-P), financiado por el Ministerio de Economía y Competitividad español y el Fondo Europeo de Desarrollo Regional (Feder), 2016-2018. 
económica global desencadenada en 2007/2008. El merecimiento aparece como una pieza clave de la economía moral del neoliberalismo, en la medida en que rige los criterios con arreglo a los cuales ha de administrarse la escasez así generada.

En este sentido, la lógica del merecimiento se contrapone a la noción del Estado benefactor, proveedor cuasi-universal de recursos: con su reducción, se produce un regreso a la arbitrariedad y la discrecionalidad, contrapuestas a una asignación de recursos con arreglo a criterios explícitos y mediante procedimientos institucionalizados, racionalizados y burocráticos. El auge del merecimiento como criterio de asignación de recursos y reconocimiento puede ser, por tanto, un retroceso del Estado moderno en favor de vínculos y obligaciones más personalizadas y clientelares.

La idea del (in)merecimiento interviene en las prácticas de los operadores jurídicos y de los representantes de instituciones estatales que asignan los recursos del bienestar. Característico de muchos análisis del merecimiento (Howe 1990; Dubois 2013, 2018; Chauvin y Garcés-Mascareñas 2014) es mostrar cómo las decisiones de estos gatekeepers no pueden explicarse como una mera aplicación del derecho positivo o de protocolos y normativas, sino que se fundamentan a menudo en categorías morales, discriminando así entre colectivos, y en consecuencia produciendo o reproduciendo formas de categorización, opresión, dominación, exclusión e inferiorización. Esto es lo que analiza Sabaté Muriel en el caso de las interpretaciones morales que los empleados bancarios realizan sobre la noción legal de "deudores de buena fe" en los procesos de desahucio, así como el análisis de Endara Rosales sobre las interpretaciones que hacen los operadores jurídicos del concepto legal de "capacidad de gobernarse a sí mismo" en los procesos de incapacitación de personas con discapacidad intelectual. El texto de Bofill-Poch y Márquez Porras también trata de cómo los litigantes y operadores jurídicos en conflictos laborales judicializados manejan juicios morales por encima incluso de las propias categorías jurídicas.

Como ya se ha apuntado, el auge del lenguaje del merecimiento se ha relacionado también con la progresiva retirada del Estado en el campo de la provisión de bienestar, un proceso coincidente, en el ámbito de las representaciones sociales, con la aparición y refuerzo de estereotipos que presentan a ciertos colectivos sociales como tendentes a depender de subsidios y transferencias de recursos públicos sin reunir las condiciones que los harían merecedores de ellos (Watkins-Hayes y Kovalsky 2016). Según esta perspectiva, la redistribución, que hasta entonces se había regido por criterios burocráticos y sobre premisas universalistas, adquiere arbitrariedad con la emisión de juicios de merecimiento: la asignación de recursos se produce con arreglo a criterios que pueden ser más discrecionales, menos fiscalizables. Esto ocurre de forma especialmente visible en los casos en que la provisión de welfare ya no se produce desde agencias estatales, sino que se externaliza a organizaciones no gubernamentales o del ámbito 
de la beneficencia. En estos casos, en lugar de asumirse una responsabilidad estatal en la provisión de recursos sobre la base de unos derechos reconocidos, la asignación se basa en el voluntarismo, el altruismo y la falta de garantías, a partir de una determinada definición de la necesidad y el merecimiento. Las organizaciones caritativas ya no atienden a ciudadanos con derechos, sino a beneficiarios o usuarios definidos como necesitados y merecedores de ayuda.

Mediante los mecanismos aquí descritos, se establece y reproduce un orden meritocrático en el que quienes aspiran a acceder a recursos deben ser en primer lugar reconocidos como merecedores de los mismos, para lo cual han de utilizar determinadas estrategias en sus interacciones con los gatekeepers (Dubois 2018; Howe 1990), al tiempo que aceptan verse sometidos a formas de escrutinio y control institucional que revelan la dimensión punitiva y disciplinadora del propio régimen meritocrático (Wacquant 2009).

\section{EL MERECIMIENTO Y LOS LENGUAJES DE LA INJUSTICIA}

Aunque a veces se utilicen como términos intercambiables, es justamente la diferencia entre deservingness (merecimiento) y entitlement (derecho) lo que confiere al primero su relevancia teórica (Feldman y Skow 2016). Una persona o grupo puede tener derechos y (desde la perspectiva de otros) no merecerlos, de la misma manera que puede no tener derecho y creer merecerlo. Suelen producirse, por tanto, una serie de solapamientos y contradicciones entre el derecho positivo y el merecimiento en términos morales, lo que da pie, a su vez, a la tensión entre legalidad y legitimidad a menudo invocada por los sujetos, ya sea de manera individual, ya sea organizándose colectivamente en movimientos sociales.

Un juicio sobre el merecimiento de alguien puede ser un juicio sobre un entitlement. En ese sentido, constituye un mensaje dirigido a la institución a la que se reclama incluir o excluir a alguien de la cobertura de un derecho. Pero también puede pretender la legitimación a posteriori de una decisión de asignación de recursos ya tomada. En ese caso, la aspiración del emisor del juicio es la conservación del status quo, y no la conquista de nuevos derechos positivos.

A diferencia de una literatura filosófica que intenta encontrar criterios de merecimiento (desert) universalmente válidos, una especie de principios morales transcendentales, el merecimiento en la vida social actúa de manera extremadamente situada, en un triple sentido. En primer lugar, situada en función de lo que Chauvin y Garcés-Mascareñas (2014) llaman los "marcos del merecimiento". Con ello se refieren a que los criterios de merecimiento que se esgrimen para legitimar el acceso a unos derechos, a menudo cambian de una situación social a otra en función de cuál sea el frame o el tipo de argumento que pueda encontrar más receptividad para lograr un objetivo concreto, incluso pudiendo entrar en tensión con los argumentos utilizados en otra situación. 
En segundo lugar, el merecimiento opera de manera situada en función del contexto de enunciación. Los relatos sobre el (in)merecimiento tienen un significado interactivo, que tiene que ver no solo con lo que se quiere denunciar o defender, sino con lo que Goffman (1987) llamaba impression management, es decir, con el efecto que se quiere lograr sobre el interlocutor (tal como lo analiza Howe 1990, por ejemplo). Siempre hay un destinatario explícito o implícito cuando se hace un juicio sobre el (in)merecimiento de alguien, y lo que se quiere conseguir con ello en muchos casos es tanto una forma de autopresentación como una demanda sobre el merecimiento de los otros. La particularidad de una mirada etnográfica sobre el merecimiento es precisamente incorporar esta significación interactiva.

Por último, los juicios de merecimiento pueden ser sobre uno mismo (ingroup) o sobre otros (outgroup). Estos últimos serían juicios heterónomos, y a menudo son emitidos por quienes detentan el poder de decidir sobre la asignación de recursos (gatekeepers), pero también por la gente corriente que opta a recursos escasos, que entran en comparaciones para determinar quién es más o menos merecedor, como ilustra el caso de García Curado sobre los complejos criterios que manejan los trabajadores de la construcción para justificar su merecimiento del puesto de trabajo.

Así pues, una ventaja ofrecida por el análisis en términos de (in)merecimiento es que ilumina principios ideológicos históricamente contingentes invocados por actores sociales en distintas posiciones o pertenecientes a distintos grupos. A menudo, la gente percibe una situación como un agravio porque cree que su mérito individual o su pertenencia a una determinada categoría social (definida en términos morales) le habilita para merecer mejor suerte, tanto en términos de acceso a recursos materiales (justicia distributiva) como en términos de reconocimiento simbólico.

En el campo de la (re)distribución material, el estallido de la crisis económica global en 2007/2008 legitimó discursos acerca de la escasez y la consiguiente necesidad de aplicar políticas de austeridad bajo una lógica de "suma cero", según la cual las partidas presupuestarias que son asignadas a una finalidad o a un colectivo han de ser detraídas de otro. Así lo muestra el texto de Aramburu sobre las ideas de sectores de la clase obrera acerca de la redistribución interterritorial, o el texto de García Curado sobre las diferentes legitimaciones que manejan los trabajadores de la construcción sobre el "derecho al trabajo”. Esto sitúa a distintos grupos sociales en competición por los recursos, y aquellos que más dependen de la provisión pública aparecen como sospechosos de no merecer lo que reciben. Como resultado, se observan procesos de categorización bajo criterios de merecimiento, así como una inferiorización de ciertos individuos y grupos como "no merecedores".

Frente a esta deriva, de la sociedad también emergen demandas de incondicionalidad o universalidad de la provisión, el apoyo o el reconocimiento. Esto 
último se traduce a menudo en términos de inclusión social, de dignidad o de "derecho a tener derechos" de manera más general, como se advierte, por ejemplo, en la contraposición entre migrantes económicos y refugiados políticos (Fassin 2005; Chauvin y Garcés-Mascareñas 2014). En algunos casos, las reclamaciones de reconocimiento e inclusión dan pie a la propuesta de (re)instaurar políticas públicas más universalistas o incondicionales, como la renta básica, o de acabar con dinámicas de exclusión de ciertos colectivos, considerados como no ciudadanos y por tanto no "elegibles" para acceder a ciertos derechos, como es el caso de los extranjeros en situación de ilegalidad.

Algunas etnografías confrontan esto con la incondicionalidad del apoyo proporcionado por las redes de parentesco $u$ otras redes sociales informales de naturaleza semejante, como en el caso de las personas sobreendeudadas que analiza Sabaté Muriel. La confrontación entre ambas lógicas revela cómo los lenguajes y lógicas del merecimiento colisionan frontalmente con la reciprocidad en términos de obligación moral, en lo que podemos considerar una tensión entre las economías morales de la reciprocidad y del neoliberalismo.

Según Feldman y Skow (2016), el merecimiento es un juicio en el que se hace una valoración del acceso de alguien a un derecho que puede estar basada en lo que ese alguien es o en lo que ese alguien hace. Así, la identidad y el comportamiento del sujeto se vuelven fuentes de (des)legitimación del acceso de alguien a un derecho (entitlement). En el caso analizado por Aramburu, el desmerecimiento no procede tanto de la asignación de una identidad grupal como de su inadecuación a lo prescrito para el sujeto normativo moderno, es decir, independiente, productivo, etc. Cobb (1973) también habla de cómo, en sociedades capitalistas como la norteamericana, el mérito (worthiness) y la superioridad moral (self-righteousness) que se auto-atribuyen las personas varía en función de su "utilidad o legitimidad social", entendidas como su aportación a la producción. Esto resulta en jerarquías como las que existen entre trabajadores cualificados y no cualificados, breadwinners y amas de casa, etc. Todo ello en consonancia con un sistema meritocrático en el que cada individuo se ve como responsable de sus acciones y su destino.

Finalmente, hablar de la justicia en términos de merecimiento frecuentemente implica reactivar y reproducir categorías de clasificación social preexistentes, ya sean raciales, étnicas, de clase, etc. Esta aplicación de categorías parte de la construcción de una alteridad por oposición a un "nosotros", y a menudo implica la aplicación de estereotipos y prejuicios. Nos encontramos ante lo que Anthony Cohen (1985) denomina "comunidades simbólicas", es decir, categorías cognitivas de clasificación entre "nosotros" y los "otros". Para Cohen, la comunidad simbólica es un repertorio de significados y un referente identitario que funciona independientemente de su base estructural: "La comunidad existe en la mente de sus miembros" (1985: 98). Pero, a diferencia de Cohen, creemos que la comunidad simbólica no solo puede funcionar como 
una categoría clasificatoria, que marca la pertenencia a un grupo con el cual nos identificamos, sino que también puede tener un carácter performativo: crea realidad, condiciona el comportamiento, genera prescripciones y expectativas, se utiliza para justificar acciones. Cuando lo que se prescribe para este grupo (simbólico) de pertenencia es una economía moral de la reciprocidad, podemos hablar de una "comunidad moral". Así, las "comunidades morales" de los sujetos son "the group for which they express concern about their living conditions", en expresión de Crepaz (2008: 1 16), y por tanto demarcan el perímetro de grupos (simbólicos) en el seno de los cuales se prescriben determinadas obligaciones morales, que se suspenden más allá de los confines de dichas comunidades. Si bien muchas veces estas comunidades morales remiten a comunidades simbólicas preexistentes, en otras ocasiones los procesos y conflictos sociales generan comunidades morales de nueva creación. Estas construcciones grupales o comunitarias dan como resultado un orden de estatus construido y/o reproducido sobre la base del (in)merecimiento.

Así pues, el objetivo del presente dossier es prestar atención a los usos sociales del merecimiento en distintos casos etnográficos, identificando las implicaciones morales implícitas en la emisión de juicios al respecto. En cada uno de los casos, analizaremos la legitimación del acceso a los derechos (entitlements) y las percepciones de injusticia, así como formas imaginadas o reales de lucha contra ellas. Desde el punto de vista de la teoría de la justicia, el análisis del lenguaje del merecimiento nos permite ver cómo se imbrican dos lógicas o dimensiones de la justicia que muchas veces se han visto como lenguajes alternativos; nos referimos a los campos del reconocimiento y de la distribución. En el habla coloquial, tanto las injusticias de distribución, que afectan a la asignación de recursos, como las de reconocimiento, que tienen que ver con la idea de dignidad o con el "derecho a tener derechos", suelen ser señaladas recurriendo a la idea del merecimiento. En ese sentido, el concepto de merecimiento nos permite sortear análisis dicotómicos del tipo distribución-reconocimiento, dimensiones de la justicia que en las situaciones etnográficas concretas suelen estar más mutuamente imbricadas de lo que muchas veces se asume desde posiciones teóricas e ideológicas. De hecho, a menudo las demandas distributivas se realizan desde una posición que reclama un determinado reconocimiento moral. En la medida en que la noción de merecimiento ilumina los aspectos morales implicados en la provisión (pública o privada) de bienestar y la administración de justicia, así como la (des)responsabilización de las élites y las instituciones públicas al respecto, se trata de explorar su utilidad para analizar la producción y reproducción de desigualdades sociales y las dinámicas de inclusión y exclusión social, así como las luchas por la justicia social en sus distintos grados de institucionalización. 


\section{BIBLIOGRAFÍA}

CHAUVIN, Sébastien, y Blanca GARCÉS-MASCAREÑAS, 2014, "Becoming less illegal: deservingness frames and undocumented migrant incorporation”, Sociology Compass, 8 (4): 422-432.

COBB, Jonathan, 1973, "Afterword", en R. Sennett y J. Cobb, The Hidden Injuries of Class. Nueva York, Vintage Books, 263-271.

COHEN, Anthony P., 1985, The Symbolic Construction of Community. Chichester, Ellis Horwood.

CREPAZ, Markus M.L., 2008, Trust beyond Borders: Immigration, the Welfare State, and Identity in Modern Societies. Ann Arbor, MI, The University of Michigan Press.

DUBOIS, Vincent, 2013, "Institutional order, interaction order and social order: administering welfare, disciplining the poor", disponible en < https://halshs.archives-ouvertes.fr/h alshs-00837517 > (última consulta en febrero de 2020).

DUBOIS, Vincent, 2018, El Burócrata y el Pobre: Relación Administrativa y Tratamiento de la Miseria. València, Institució Alfons el Magnànim.

FASSIN, Didier, 2005, "Compassion and repression: the moral economy of immigration policies in France", Cultural Anthropology, 20 (3): 362-387.

FELDMAN, Fred, and Brad SKOW, 2016, "Desert", en Edward N. Zalta (coord.), The Stanford Encyclopedia of Philosophy (Winter 2016 Edition), disponible en < https://plato.stanfor d.edu/archives/win2016/entries/desert/ > (última consulta en febrero de 2020).

GOFFMAN, Erving, 1987, La Presentación de la Persona en la Vida Cotidiana. Buenos Aires, Amorrortu.

HOWE, Leo, 1990, Being Unemployed in Northern Ireland: An Ethnographic Study. Cambridge, Cambridge University Press.

KATZ, Michael B., 1989, The Undeserving Poor: From the War on Poverty to the War on Welfare. Nueva York, Pantheon Books.

RUGgiero, Vincenzo, 2018, Los Crímenes de la Economía: Un Análisis Criminológico del Pensamiento Económico. Madrid, Marcial Pons.

SCOTT, James, 1976, The Moral Economy of the Peasant: Rebellion and Subsistence in Southeast Asia. New Haven y Londres, Yale University Press.

THOMPSON, Edward, 1971, "The moral economy of the English crowd in the eighteenth century", Past and Present, 50: 76-136.

WACQUANT, Loïc, 2009, Punishing the Poor: The Neoliberal Government of Social Insecurity. Durham, NC, Duke University Press.

WATKINS-HAYES, Celeste, y Elyse KOVALSKY, 2016, "The discourse of deservingness: morality and the dilemmas of poverty relief in debate and practice”, en D. Brady y L. M. Burton (coords.), The Oxford Handbook of the Social Science of Poverty, disponible en < https:// www.oxfordhandbooks.com/view/10.1093/oxfordhb/9780199914050.001.0001/oxford hb-9780199914050-e-10 > (última consulta en febrero de 2020).

WHYTE, David, y Jörg WIEGRATZ (coords.), 2016, Neoliberalism and the Moral Economy of Fraud. Londres, Routledge. 